Ophthalmologica 1969;158:I-IV

\title{
Contents, Vol. 158, No. 1-3, 1969
}

3rd Congress of the European Society of Ophthalmology

3. Kongreß der Europäischen Gesellschaft für Ophthalmologie

3e Congrès de la Société Européenne d'Ophtalmologie

Under the high patronage of Her Majesty the Queen of the Netherlands Amsterdam, 18-21 June, 1968

Occupational and Medicative Hazards in Ophthalmology

Edited by

J. Francois, Ghent

Secretary General of the European Society of Ophthalmology

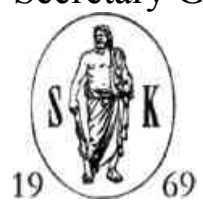

BASEL (Switzerland) S. KARGER

NEW YORK

S. Karger AG, Arnold-Böcklin-Strasse 25, 4000 Basel 11 (Switzerland)

All rights, including that oí translation into other languages, reserved

Photomechanic reproduction (photocopy, microcopy) of this book or parts

thereof without special permission of Ihe publishers is prohibited

(C)

Copyright 1909 by S. Karger AG, Basle Printed in Switzerland by Friedrich Reinhardt AG,

Basle

Inhaltsverzeichnis - Contents - Sommaire

Hauptreferate - Main Lectures - Rapports principaux

Nordmann, J. (Strasbourg):

L'ophtalmologie, sa position dans la médecine, au passé, au present

et à Tavenir 1

Roper-Hall, M. J. (Birmingham):

A Retrospective Study of Eye Injuries

Rougier, J. (Lyon):

Le traitement chirurgical des paralysies oculaires secondaires auxfractures de Torbite

Tönjum, A. M. (Oslo):

Contusion of the Eye 49

Stankovic, I. (Belgrade):

Interventions électromagnétiques répétées 57

Moutinho, H. (Lisboa):

L'extraction des corps étrangers magnétiques du cristallin transparent 64 
Heinz, K. (Innsbruck):

Ultraviolette und Sonnenstrahlung 75

Lange, J. (Gummersbach):

Chemische Schädigungen des Auges in der Arbeitsmedizin ... 95

Bahr, G. von (Uppsala):

Electrical Injuries 109

Oksala, T. (Turku):

Schädigungen durch Ultraschall am Auge 118

Blake, J. (Dublin):

Ocular Hazards in Agriculture

Grósz, I. de (Budapest):

Chemical Burns of the Eye due to Pesticides 136

Salorio, M. S. (Santiago, Espagne):

Intoxication par Talcool méthylique 141

Smith, V. H. (Birmingham):

The Prevention of Ocular Injuries in Industry

Dubois-Poulsen, A. (Paris):

La fatigue visuelle 157

Heine, A. et Kubëna, K. (Olomouc):

Maladies oculaires extemes par action directe d'éléments extérieurs 181

Grignolo, A. (Genova) et Scano, A. (Roma):

Maladies professionnelles oculaires en rapport avec les vols atmosphé-riques et spatiaux 191

Nelemans, F. A. (Leidschendam):

Treating the Patient a Threat for his Eyes 208

Schroder, K. E. (Gießen):

Schädigungen des vorderen Augenabschnittes durch Medikamente . 218

Crews, S. J. (Binmingham):

Some Aspects of Retinal Drug Toxicity 232

B. Bruckner

R. (Basel):

Frühdiagnose medikamentöser Schäden von Netzhaut und Sehnerv 245 